\title{
CASE OF POLYCYSTIC OVARIAN TUMOUR COMPLICATED BY ASCITES.
}

\author{
BY R. J. KINKEAD, M.D.; \\ Professor of Obstetrics, Queen's College, Galway; \\ Physician and Gynæcologist to the Galway Hospital. \\ [Read in the Section of Obstetrics, Mry 26, 1899.]
}

Mrs. N., nullipara, aged fifty, admitted to the Galway Hospital 30th June, 1898. It was impossible to get a reliable history; she, however, asserted that she only began "to get big within the year;" that there was no enlargement of the abdomen twelve months ago ; she was sure there was no lump in either groin at the beginning, and she had been tapped three or four"times. The latter statement was confirmed by the marks of the trocar. The heart, lungs, and kidneys were healthy, but, owing to the enormous distension of the abdomen, the size and condition of the liver could not be made out: there was no bile in the urine.

The characteristic ovarian expression was absent, face not emaciated, countenance cheerful. The abdomen immensely distended, umbilicus prominent, curve to ensiform cartilage gradual, that to pubes more abrupt than generally seen in ascites, though not more so than in two cases in which I had evacuated ascitic fluid a few days previously, fluctuation very distinct all over abdomen, which was dull on percussion in front, at sides, at right flank behind, and, although not so dull as the right, yet a clear note was not given over the left: the surface of abdomen was smooth, resistance seemed equal everywhere, no nodules or tumour could be felt.

It was clear that there was ascites, and the previous tapping pointed that way. Not being satisfied that it was only ascites I determined to make an exploratory incision and proceed further if necessary. On the 2nd July I operated, assisted by Professor Brereton and Dr. M'Kelvey. The patient having passed rapidly under ether, I commenced the operation at 12 noon by making a short incision down to the peritoneum, on nicking which clear ascitic fluid gushed out in a strong stream, 
a trocar or cantula was then introduced, it, however, struck on some obstacle within. I then slit up the peritoneum to the size of the external incision and exposed a tumour apparently covered by a muscular coat with large, distended veins ramifying on its surface: the incision was then extended up to a couple of inches above the umbilicus, when it was seen that the tumour was a huge ovarian cyst, and that the muscular layer terminated below umbilictis, being triangular in shape-apex above and the base below. There were numerous adhesions to the abdominal wall, especially at site of the tappings, and also omental, some of which I separated, others had to be tied and cut.

I endeavoured to draw off contents of cyst with a Spencer Wells' trocar but failed, as before it passed in as far as the clips it struck on a resisting body inside the cyst. The patient was turned on her side and the cyst drawn up to the wound and the trocar wilhdrawn; as the contents were evacuated, the opening in the cyst was drawn well through the wound. Having emptied the cyst, the patient was turned on her back, and I slit it up and found close to the lower margin of opening a small cyst and immediately behind a very large one, which was tapped with a Spencer Wells' trocar, and as it emptied the mass was drawn by eyst forceps well up into the wound. The delivery of the cysts through the abdominal incision was taken charge of by Professor Brereton, while I separated pelvic and other adhesions; yet, when freed in front, above, below, and at the sides, it was still fixed, and it was only when I got my hand behind it that I.found it was anchored in position by four cysts projecting from it, each as big as a foetal head, situated two on each side of the spine; and it was not until I got my hand underneath and dislodged these from their positions that we were able to lift the mass of cysts out of the abdomen.

The pedicle, short and thick, sprang from left side of the uterus, which was drawn up out of pelvis: notwithstanding that it was tied in three sections, on being separated from the tumour, two considerable sized arteries spouted; one I tied, the other was secured by the continuous suture with which I overran the cut edge of the pedicle (fully three inches wide), bringing the peritoneal edges together over the cut surface. The other ovary being diseased was also removed. The peritoneum was closed by a continuous suture of fine silk, and the abdominal wound by interrupted sutures of silkworm-gut. 
At the termination of the operation the woman's condition was alarming; $\frac{l}{60}$ gr. strychnin and three syringefuls of ether were injected hypodermically: she was placed in a warm bed, the foot of the bedstead elevated, and an enema of beef tea with an ounce of brandy was administered. She rallied rapidly, and except for a stitch abscess convalescence was uneventful.

We were not able to catch and measure the ascitic fluid and that in the outer cyst; the fluid drawn from the inner cyst filled a metal slop bucket.

Immediate operation was necessary for the enormous distension. Another laparotomy case occupied the ward used for such patients. I was therefore forced to operate in one of the ordinary wards in which were various medical cases; this, of course, added considerably to the anxiety. Happily, however, she progressed as favourably as if she had been isolated.

DR. SMYr.Y expressed surprise that tapping should have been resorted to by those having charge of the case before Dr. Kinkead. He was of opinion that any medical man who tapped an ovarian cyst should be liable to prosecution for malpractice. The Spencer Wells trocar was one of those instruments which were quite useless, and it was impossible to keep it aseptic. The muscular coat which covered the front of the tumour, he thought, might hare been the broad ligament.

Dr. KNoTr related the case of a young woman suffering from a rapidly growing tumour. She was tapped, as they were very chary of performing ovariotomy in those days. The patient died finally of slow suffocation, and the necropsy, at which he was present, revealed a polycystic ovarian tumour extending up into the thorax.

Dr. F. W. KIDD said he was called into consultation in a case of a very large ovarian cyst, and to relieve dyspnœa and to benefit the puerperium, the patient having been recently delivered, he advocated tapping, cautioning, however, the medical man in attendance that this was only a palliative measure. Orer 30 pints of fluid characteristic of an ovarian cyst were withdrawn. He was able to state that the cyst did not fill again, and that the patient had completely reeorered. 